\title{
The Effect of Financial Conflict of Interest, Disclosure Status, and Relevance on Medical Research from the United States
}

\author{
Deepa V. Cherla, MD ${ }^{1,2}$, Cristina P. Viso, MD ${ }^{7}$, Julie L. Holihan, MD, MS ${ }^{1,2}$, Karla Bernardi, MD ${ }^{1,2,3}$, \\ Maya L. Moses, MDㄱ, Krislynn M. Mueck, $M D^{1,2}$, Oscar A. Olavarria, $M D^{7}$, \\ Juan R. Flores-Gonzalez, MD' , Courtney J. Balentine, MD', Tien C. Ko, MD' , Sasha D. Adams, MD', \\ Claudia Pedroza, $P h D^{1,5}$, Lillian S. Kao, MD, $M S^{1,2}$, and Mike K. Liang, $M D^{1,2}$
}

\begin{abstract}
'Department of Surgery, McGovern Medical School, University of Texas Health Science Center at Houston, Houston, TX, USA; ${ }^{2}$ Center for Surgical Trials and Evidence-Based Practice (C-STEP), McGovern Medical School, University of Texas Health Science Center at Houston, Houston, TX, USA; ${ }^{3}$ Department of Surgery, General Surgery Clinical Research Fellow, Houston, TX, USA; ${ }^{4}$ Department of Surgery \& Institute for Cancer Outcomes and Survivorship, University of Alabama at Birmingham, Birmingham, AL, USA; ${ }^{5}$ Center for Clinical Research and Evidence-Based Medicine, McGovern Medical School, University of Texas Health Science Center at Houston, Houston, TX, USA.
\end{abstract}

BACKGROUND: Financial interactions between industry and healthcare providers are reportable. Substantial discrepancies have been detected between industry and selfreport of these conflicts of interest (COIs).

OBJECTIVE: Our aim was to determine if authors who fail to disclose reportable COI are more likely to publish findings that are favorable to industry than authors with no COI.

DESIGN: In this blinded, observational study of medical and surgical primary research articles in PubMed, 590 articles were reviewed.

MAIN MEASURES: Reportable financial relationships between authors and industry were evaluated. COIs were considered to have relevance if they were associated with the product(s) mentioned by an article. Primary outcome was favorability, defined as an impression favorable to the product(s) discussed by an article and determined by 3 independent, blinded clinicians for each article. Primary analysis compared Incomplete Self-Disclosure to No COI. Two-level multivariable mixed-effects ordered logistic regression was used to assess factors associated with favorability.

KEY RESULTS: A 69\% discordance rate existed between industry and self-report in COI disclosure.

When authors failed to disclose COI, their conclusions were more likely to favor industry partners than authors without COI (favorable ratings $73 \%$ versus $62 \%$, RR 1.18 , $p=<0.001$ ). On univariate (any COI $74 \%$ versus no COI $62 \%$, RR 1.11, $p=<0.001$ ) and multivariable analyses, any COI was associated with favorability.

CONCLUSIONS: All financial COIs (disclosed or undisclosed, relevant or not relevant, research or nonresearch) influence whether studies report findings favorable to industry sponsors.

Electronic supplementary material The online version of this article (https://doi.org/10.1007/s11606-018-4784-0) contains supplementary material, which is available to authorized users.

Received March 20, 2018

Revised August 31, 2018

Accepted November 21, 2018

Published online January 2, 2019
KEYWORDS: ethics; medical education-professionalism; research Design.

J Gen Intern Med 34(3):429-34

DOI: $10.1007 / \mathrm{s} 11606-018-4784-0$

(c) Society of General Internal Medicine 2018

\section{INTRODUCTION}

Conflicts of interest (COIs) increase potential for biased judgment $^{1}$ and have historically led to considerable suspicion of study findings. Multiple studies and papers have alluded to the pervasive nature of financial relationships between clinicians and manufacturers as well as reflected the increased scrutiny of such interactions. ${ }^{2}$ COIs are sources of bias ${ }^{3}$ defined by the Institute of Medicine (IOM) as "circumstances that create a risk that professional judgment or actions regarding a primary interest will be unduly influenced by a secondary interest." 4 They may be financial or non-financial. ${ }^{4,5}$ Case reports and original research support an influence of COIs on study conception, data collection, and writing. ${ }^{6,7}$ A study claiming that exposure to asbestos-containing roofing products was within safe limits had to be withdrawn following criticism concerning the approving editor's associations with the asbestos industry. ${ }^{8}$ An industry-sponsored drug study provided a deceptively favorable profile of the medication by reporting 12-month outcomes as if they occurred at 6 months. ${ }^{9,}{ }^{10}$ In 2001, an industry-sponsored study claimed the antidepressant Paroxetine to be "generally well tolerated and effective" in depressed adolescents. A reanalysis of the data by independent researchers, published in 2015, concluded that Paroxetine was actually neither safe nor effective in this population. ${ }^{11}$ Independent organizations' reviews of studies written by a physician-scientist who received funding from organizations critical of genetically modified organisms concluded that the researcher's statements were actually unsupported by his data. $^{12}$

Until recently, reporting of COIs has relied almost entirely on author self-disclosure. In an attempt to improve 
transparency, the Physician Payments Sunshine Act, passed in 2010, now requires manufacturers of drugs, medical devices, and biological products to report all payments to clinicians having a National Provider Identifier (NPI). ${ }^{13}$ These financial transactions are reported to the public through the Open Payments Database (OPD). ${ }^{14}$ In 2015, 11.90 million transactions were reported on the OPD, amounting to a total of $\$ 7.52$ billion dollars. ${ }^{3,14}$

While COIs have contributed to the skepticism of published findings and clinical recommendations, ${ }^{15-17}$ few quantitative, scientific evidence exist concerning the global impact of disclosed and undisclosed financial COIs on study conclusions. We hypothesized that (1) articles with COI undisclosed by authors as opposed to articles with no COI are more likely to provide favorable impressions of discussed article product(s); (2) the presence of COIs, regardless of disclosure status, increases favorability; and (3) as the amount of total payments to the authors of an article increases, the favorability portrayed by the article toward a discussed product also increases.

\section{MATERIALS AND METHODS}

This was a blinded, observational study of previously published primary research articles. Strengthening the Reporting of Observational Studies in Epidemiology Statement (STROBE) guidelines were followed. ${ }^{18}$ PubMed was searched for articles in multiple randomly selected specialties as defined by the Accreditation Council for Graduate Medical Education (Supplementary Table 1) ${ }^{19}$ Articles were retrieved in reverse chronological order. Inclusion criteria included an acceptance date from 2013 to 2016 (corresponding to dates for which OPD data was available), at least 1 product listed in the title or abstract, at least 1 author with an NPI number (to qualify for inclusion in the OPD), and availability of an accompanying abstract. Self-disclosure statements were obtained from the full text of each article. The industry-reported COI for every author was obtained from the OPD. In accordance with the IOM, we defined payments listed in the OPD as $\mathrm{COI}$ if they were reported as honoraria, gifts, payments for research or consulting, compensation for serving as faculty or speaker, company ownerships/partnerships, and food/travel expenses greater than or equal to $\$ 5000 .^{3}$ The number of industry-reported COIs, total payments received by all authors in the 1 year preceding a manuscript's acceptance date, and the total number of interactions with industry (equal to the number of interactions recorded on the OPD) between authors of each manuscript and companies were also recorded. Two reviewers assessed relevance of self-reported and industry-reported COIs to the discussed product(s) in an article. Relevance was determined by performing an Internet search of all products produced by each named company and comparing these products to those described by each article. If a company produced the products directly mentioned by an article or produced competing products, that company was considered to be a relevant COI. Relevant COIs were determined to be either disclosed by manuscript authors (self-disclosed), by the OPD (non-self-disclosed), or both (full disclosure). The reviewers were blinded to abstract authors and their COIs as well as disclosure status of the conflicts.

Manuscripts were categorized into 4 disclosure categories: ${ }^{3}$ Full Disclosure, Incomplete Self-Disclosure (authors failed to disclose all COIs described on the OPD), Incomplete Industry Disclosure (industry did not disclose all COIs self-disclosed by authors), and No COI. Incomplete Self-Disclosure was also subdivided into Partial and No Disclosure categories. Partial Disclosure included all articles for which authors listed some but not all COIs reported in the OPD. No Disclosure included all articles for which authors listed no COI but for whom the OPD had listed COI. Disclosure categories were determined by comparing all self-disclosed COIs versus all OPDdisclosed COIs for each manuscript. Therefore, if 1 author of a study disclosed Conflict A for whom the OPD also disclosed only Conflict A, and a second author of the same study disclosed nothing but for whom the OPD disclosed Conflict B, this manuscript would have been coded as Incomplete Self-Disclosure. If, on the other hand, the second author was found to also be associated with Conflict A per the OPD, this manuscript would have been categorized as Full Disclosure. We considered disclosure categories on the manuscript rather than on the author level to reflect the collaborative nature of manuscript-writing; all authors' inputs, as well as COIs, were considered important for each paper's overall message and COI disclosure status.

Primary outcome was favorability defined as the impression an article provides of its subject matter. A panel of 3 independent, blinded clinicians reviewed and ranked the impression each article conveyed of product(s) within the article title on a 1-5 Likert scale in which "1" and "2" were "Unfavorable," "3" was neutral, and " 4 " and " 5 " were "Favorable." The reviewers were blinded to the article authors, institution, and COI. Article favorability was oriented based on specific author COI. For example, to address competitive company COI, if an article whose authors examined a product sold by Company B but who were affiliated with a competitor Company A, the ordinal response was flipped to adjust for such affiliations. If an article discussed more than 1 product, each reviewer also answered separate questions, or Likert scales, relating to the article's specific favorability for each product. Therefore, the vendors of all products, including those favorably, neutrally, or unfavorably portrayed in the papers, were compared with the self-declared sources of support for the authors. Secondary outcomes included inter-reliability between the favorability scores of each manuscript question (3 favorability scores per manuscript), number of COIs, number of interactions, amount of total payments, and amount of research- and non-researchrelated funding. 
Data Availability. The data reported in the paper can be made available by contacting the corresponding author.

\section{STATISTICAL ANALYSIS}

Univariate statistical analysis was performed for favorability using chi-square tests. One-way random-effect intraclass correlation was calculated to determine inter-rater reliability between the favorability scores of each manuscript question. Primary analysis compared Incomplete Self-Disclosure to No COI. Secondary analyses compared articles with any COI and all subgroups $(n=4)$ to those with No COI using chi-square or Kruskall-Wallis test. Due to multiple testing of the secondary analyses, we performed a Bonferroni correction ( $p<0.05 /$ number of analyses $=5$ ) and considered $p<0.01$ as significant.

A two-level (manuscript, question) multivariable mixedeffects ordered logistic regression model was used to assess factors associated with favorability to account for clustering on the rater- and manuscript- levels. The dependent outcome variable, favorability as an ordinal variable (1 to 5), was modeled as a function of the following independent variables: COI Disclosure Category (Full Disclosure, Incomplete Industry Disclosure, Incomplete Self-Disclosure, and No Disclosure), Relevance (yes/no), Research Payments (\$0, \$1-5000, \$5001-25,000, greater than $\$ 25,000)$, and Non-Research Payments $(\$ 0, \$ 1-$ $5000, \$ 5001-25,000$, greater than $\$ 25,000)$. Interactions were also assessed between disclosure category, relevance, and payments. Model fit was examined by comparing Akaike Information Criterion (AIC). Two sensitivity analyses including the same covariates and random effects were performed. One excluded articles with incomplete industry reporting (due to missing information in the OPD). The second one used a dichotomized favorable outcome (scores of 4 to 5 versus 1 to 3 ) in a multilevel logistic model.

Sample size was calculated by assuming a difference of $10 \%$ in favorability (assuming 50\% of studies with no COI were "favorable"), an alpha of 0.05 , and a beta of 0.20 . It was determined that 357 responses (119 manuscripts) would be needed per category (no COI and incomplete self-disclosure) for the primary study outcome. Based upon these calculations, we estimated a need to review 476 articles (4 categories $\times 119$ articles).

All statistical analyses were performed with Stata Version $14.1 \circledR$.

\section{RESULTS}

Three clinicians reviewed a total of 591 articles ( 8 overall reviewers, 3 reviewers per manuscript; total number of reviews $n=1773$ ) (Fig. 1). The mean number of questions per article was 1.2, reflecting the majority of articles describing 12 products ( 1 question, or Likert scale, per product). The estimated intra-class correlation between scores within each manuscript question was 0.621 (95\% CI, 0.581-0.659).

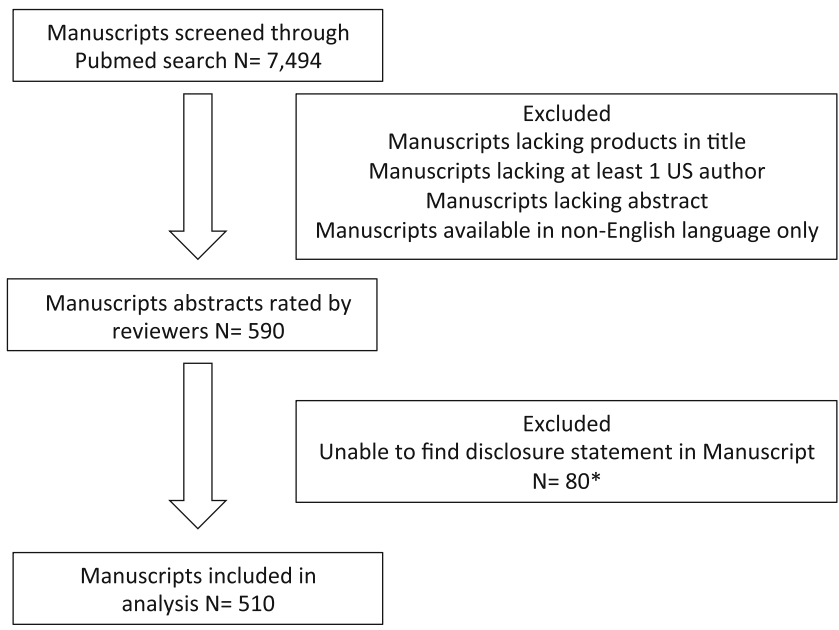

Figure 1 Flowsheet of manuscripts.

Articles with authors who did not disclose all COI as opposed to those with no COI showed increased favorability ( $73 \%$ versus $62 \%$, RR 1.17, 95\% CI 1.08-1.28, $p<0.001$, Table 1 ).

Articles with financial COI, regardless of disclosure status, had statistically significantly higher favorability than articles with no COI (74\% versus $62 \%$; RR 1.19 , $95 \%$ confidence interval $1.10-1.29, p=<0.001)$. When comparing Full Disclosure, Incomplete Self-Disclosure, and Incomplete Industry Disclosure individually to No COI (Table 1), there was a significant difference in percentage of favorable responses and a significant increase in relative risk of having a favorable response if financial COI were present per disclosure category (Table 1). On subgroup analysis, both Partial Disclosure $(n=321 / 438$, $73 \% ; \mathrm{RR}=1.18,95 \%$ CI $1.08-1.30, p<0.001)$ and No Disclosure $(322 / 444,73 \%$; RR $=1.17,95 \%$ CI $1.07-1.29$, $p<0.001)$ demonstrated increased favorability over no COI. Excluding manuscripts for which COI were determined to be not relevant to the products listed in the article titles did not significantly change the percentage of favorable responses (Table 2). Total payments, research payments, non-research payments, and number of interactions also significantly differed per COI Disclosure Category $(p<0.001$ for all, Table 3$)$.

\section{MIXED-EFFECTS MULTIVARIABLE ORDERED LOGISTIC REGRESSION ANALYSIS}

In order to assess the relationship to COI, disclosure, payment amount and type, and favorability, a regression analysis was performed. COI Disclosure Category was found to be significantly associated with favorability (Table 4). Relevance, Research, and Non-Research Payments were not found to be significant. No interaction was detected between disclosure category, relevance, and payments. Subgroup and sensitivity analyses demonstrated similar results. 
Table 1 Number of Favorable and Unfavorable Responses per COI Disclosure category

\begin{tabular}{|c|c|c|c|}
\hline & & Industry Disclosure (OPD) & \\
\hline \multirow{9}{*}{ Self-Disclosure } & \multirow{4}{*}{ Yes } & Yes & No \\
\hline & & Full Disclosure & Incomplete Industry Disclosure \\
\hline & & Favorable 52/60 (87\%) & Favorable $126 / 171(74 \%)$ \\
\hline & & Unfavorable $4 / 60(7 \%)$ & Unfavorable $19 / 171(11 \%)$ \\
\hline & \multirow{5}{*}{ No } & Relative risk $1.40(1.24-1.59), p<0.001$ & Relative risk $1.19(1.06-1.34), p=0.004$ \\
\hline & & Incomplete Self-Disclosure & No COI \\
\hline & & Favorable $643 / 882(73 \%)$ & Favorable $260 / 420(62 \%)$ \\
\hline & & Unfavorable $108 / 882(12 \%)$ & Unfavorable $100 / 420(24 \%)$ \\
\hline & & Relative risk $1.18(1.08-1.28), p<0.001$ & Relative risk reference \\
\hline
\end{tabular}

* Relative risk is the risk of favorable results when compared to no COI. Each rater provided 1 favorability score per manuscript. Each manuscript received 3 favorability scores by 3 independent blinded reviewers

This table addresses hypothesis 1 (articles with COI undisclosed by authors as opposed to articles with no COI are more likely to provide favorable impressions of discussed article product(s)) and calculating relative risk with No COI as the control performed statistical testing

\section{DISCUSSION}

Financial COI, disclosed or undisclosed, "relevant" or "irrelevant," may influence published scientific studies compared to studies with no COI. Financial support provided by relevant COI may affect results by providing authors a material incentive to favor the source of monetary support. Authors having COI not considered relevant may potentially be seeking additional or future monetary support from industry. This is the first study to correlate study favorability, data from the OPD, self-reported COI, relevance of COI, and payment amount and type into a single analysis.

There was an extremely high rate of discordance in the reporting of COI. Reasons for discordance between selfdisclosure and OPD-disclosed COI are unknown but may include the following: human error, researchers omitting COI considered not relevant to investigated topic(s), lack of awareness of COI, and errors in the OPD. Excluding articles with "irrelevant" COI did not substantially change favorability rates. While analysis of articles in the Full Disclosure category is hampered by their small number $(n=20 / 511$, or just $4 \%$ of the articles sampled), the high favorability observed within this category may be explained by the high number of articles perhaps being explicitly funded by 1 particular company. The lower number of median COI supports this for the Full Disclosure Category than for the other disclosure groups (Table 3). It may be that these authors had a clear and strong relationship with industry when designing and writing the study. However, our multivariable analysis suggests that favorability rates are increased regardless of concordance of disclosure between authors and the OPD. Authors who "over-disclose" ties also led to industryfavored publications; this represented only a small percentage, $11 \%$, of manuscripts. We attributed the bulk of this group to industry or human error which is a more realistic estimate as compared to the group of authors who failed to selfdisclose (58\% of manuscripts). In addition, some authors who "over-disclose" could have relationships with the industry outside of the 1-year preceding article acceptance for publication. Once the OPD has accumulated multiple years of data, future studies should assess the duration of effect of financial transactions on favorable reporting.

While articles with financial COI demonstrate a high favorability rate, articles lacking any financial COI still showed strikingly high favorability toward industry and products, suggesting that factors other than monetary relationships affect publications or that total food/travel expenses less than $\$ 5000$ may also affect the writing and research styles of physicianscientists. The actual probability of "true" studies has been posited to rely on a combination of power, ratio of true to nottrue relationships, and bias; well-powered epidemiological studies, per this logic, may have only a $20 \%$ chance of being true. ${ }^{17}$ In this study, articles with no financial COI were

Table 2 Number of Favorable Responses per COI Disclosure Category Sorted by COI Relevance

\begin{tabular}{|c|c|c|c|c|c|c|}
\hline$N=$ number of manuscripts & $\begin{array}{l}\text { Full } \\
\text { Disclosure } \\
(n=20)\end{array}$ & $\begin{array}{l}\text { Incomplete } \\
\text { Self-Disclosure: } \\
\text { Partial Disclosure } \\
(n=144)\end{array}$ & $\begin{array}{l}\text { Incomplete } \\
\text { Self-Disclosure: } \\
\text { No Disclosure } \\
(n=150)\end{array}$ & $\begin{array}{l}\text { Incomplete } \\
\text { Industry } \\
\text { Disclosure } \\
(n=57)\end{array}$ & $\begin{array}{l}\text { Control: } \\
\text { No COI } \\
(n=140)\end{array}$ & $p$ value \\
\hline Manuscripts with relevant COI & $15(75 \%)$ & $132(92 \%)$ & $94(63 \%)$ & $47(83 \%)$ & - & $<0.001$ \\
\hline $\begin{array}{l}\text { Favorable responses for } \\
\text { manuscripts with relevant COI }\end{array}$ & $40 / 45(89 \%)$ & $282 / 396(71 \%)$ & $192 / 276(70 \%)$ & $104 / 141(74 \%)$ & $260 / 420(62 \%)$ & $<0.001$ \\
\hline $\begin{array}{l}\text { Favorable responses for } \\
\text { manuscripts with only irrelevant COI }\end{array}$ & $11 / 15(73 \%)$ & $39 / 42(93 \%)$ & $130 / 168(77 \%)$ & $22 / 30(73 \%)$ & $100 / 420(24 \%)$ & $<0.001$ \\
\hline
\end{tabular}

Each manuscript had 3 responses from three independent, blinded reviewers. Each rater provided 1 favorability score per manuscript. Each manuscript received 3 favorability scores by 3 independent blinded reviewers

This table addresses hypothesis 1 (articles with COI undisclosed by authors as opposed to articles with no COI are more likely to provide favorable impressions of discussed article product(s)), hypothesis 2 (the presence of COI, regardless of disclosure status, increases favorability), and calculating chi-square with No COI as the control performed statistical testing 
Table 3 Univariate Analysis of Factors Stratified by COI Disclosure Category

\begin{tabular}{|c|c|c|c|c|c|c|}
\hline & $\begin{array}{l}\text { Full } \\
\text { Disclosure }\end{array}$ & $\begin{array}{l}\text { Incomplete } \\
\text { Self-Disclosure: } \\
\text { Partial Disclosure }\end{array}$ & $\begin{array}{l}\text { Incomplete } \\
\text { Self-Disclosure: } \\
\text { No Disclosure }\end{array}$ & $\begin{array}{l}\text { Incomplete } \\
\text { Industry } \\
\text { Disclosure }\end{array}$ & $\begin{array}{l}\text { Control: } \\
\text { No COI }\end{array}$ & $p$ value \\
\hline $\begin{array}{l}\text { Number of } \\
\text { interactions } \\
\text { (median, IQR) }\end{array}$ & $4(1-29)$ & $20(5-58)$ & $5(2-18)$ & NA* & - & $<0.001$ \\
\hline $\begin{array}{l}\text { Number of COI } \\
\text { (median, IQR) }\end{array}$ & $1(1-2)$ & $6(3-10)$ & $2(1-3)$ & $2(1-4)$ & - & $<0.001$ \\
\hline $\begin{array}{l}\text { Total payments } \\
\text { (median, IQR) }\end{array}$ & $\begin{array}{l}11,603 \\
(2813-62,408)\end{array}$ & $\begin{array}{l}89,310 \\
(17,825-353,176)\end{array}$ & $\begin{array}{l}18,400 \\
(4141-75,081)\end{array}$ & $\mathrm{NA}^{*}$ & - & $<0.001$ \\
\hline $\begin{array}{l}\text { Research payments } \\
\text { (median, IQR) }\end{array}$ & $0(0-1375)$ & $18,897(0-128,201)$ & $775(0-20,461)$ & $\mathrm{NA}^{*}$ & - & $<0.001$ \\
\hline $\begin{array}{l}\text { Non-research } \\
\text { payments } \\
\text { (median, IQR) }\end{array}$ & $\begin{array}{l}11,265 \\
(1210-41,887)\end{array}$ & $\begin{array}{l}29,767 \\
(4358-94,817)\end{array}$ & $\begin{array}{l}7002 \\
(500-28,133)\end{array}$ & $\mathrm{NA}^{*}$ & - & $<0.001$ \\
\hline
\end{tabular}

*For incomplete industry disclosure, there was no information in OPD so this information could not be completely or accurately captured This table does not address the three core hypotheses and statistical testing was through Kruskal-Wallis test

determined to have a favorability of $62 \%$, suggesting that potentially $40 \%$ of favorability results from non-financial sources of COI. This could include individual researcher biases driven by career pressures, mentorship, pride, etc., or systems-based biases such as publication bias of positive results. However, these could also be related to non-financial COI such as relationships physicians form with industry apart from financial transactions. Our multivariable analysis begins to quantify these other variables by evaluating, in addition to payments and disclosure status, the actual relevance of the COI to the article topic. Additional variables that we did not evaluate in our hierarchical linear regression model include career position and aspirations of physician-scientists, choice of researchers to perform studies on experiments likely to have positive outcomes, and publication bias at the level of scientific meetings and journals. Future studies should evaluate the influence of these additional non-financial COI on article favorability.

Our finding, that both disclosed and undisclosed financial COI influence study findings, is supported by major historical cases. In what has been dubbed "the most damaging medical hoax of the last 100 years," Dr. Andrew Wakefield posited a connection between the measles-mumps-rubella vaccine and autism-enterocolitis. Editors of the journal in which the article had been published later discovered that Dr. Wakefield had failed to disclose that he had received approximately $\$ 670,000$ in compensation as a consultant to lawyers of parents of children allegedly harmed by the vaccine and that he was an inventor on a patent for a new vaccination. Despite 11 of the 12 coauthors subsequently issuing a formal retraction of the manuscript's claims and the General Medical Council Fitness to Practice Panel recommending revocation of Dr. Wakefield's license to practice medicine in England, measles outbreaks began to occur in London and rapidly spread to Scotland and Ireland following claims made by the criticized paper. ${ }^{20}$ These incidents may have been prevented had Dr. Wakefield accurately disclosed his COI.

Limitations of this study include the inability to find disclosure statements for $14 \%$ of manuscripts. While high, this number is actually smaller than those cited by other studies. ${ }^{21}$ The favorability of these excluded manuscripts was only found to significantly differ from manuscripts with no COI

Table 4 Mixed-Effects Multivariable Ordinal Logistic Regression Analysis

\begin{tabular}{|c|c|c|c|}
\hline Variable & Odds ratio & $p$ value & 95\% CI \\
\hline \multicolumn{4}{|l|}{ COI category } \\
\hline Full Disclosure & 8.65 & 0.001 & $2.46-30.44$ \\
\hline Incomplete Industry Disclosure & 3.61 & 0.003 & $1.53-8.51$ \\
\hline Incomplete Self-Disclosure (Partial) & 4.14 & 0.004 & $1.58-10.82$ \\
\hline Incomplete Self-Disclosure (None) & 4.17 & 0.002 & $1.71-10.16$ \\
\hline Relevant & 0.65 & 0.14 & $0.37-1.15$ \\
\hline \multicolumn{4}{|l|}{ Research payments } \\
\hline$\$ 1-5000$ & 0.48 & 0.05 & $0.20-0.98$ \\
\hline$\$ 5001-25,000$ & 1.37 & 0.41 & $0.65-2.88$ \\
\hline$>\$ 25,000$ & 0.86 & 0.59 & $0.49-1.49$ \\
\hline \multicolumn{4}{|l|}{ Non-research payments } \\
\hline$\$ 1-5000$ & 0.69 & 0.37 & $0.31-1.55$ \\
\hline$\$ 5001-25,000$ & 0.72 & 0.41 & $0.33-1.58$ \\
\hline$>\$ 25,000$ & 0.79 & 0.51 & $0.38-1.62$ \\
\hline
\end{tabular}

This table addresses hypothesis 1 (articles with COI undisclosed by authors as opposed to articles with no COI are more likely to provide favorable impressions of discussed article product(s)), hypothesis 2 (the presence of COI, regardless of disclosure status, increases favorability), hypothesis 3 (as the amount of total payments to the authors of an article increase, the favorability portrayed by the article toward a discussed product also increases), and was performed using a mixed-effects multivariate ordinal logistic regression 
(Fig. 1, 181/240, 75\%, $p=0.14$ ). The authors of this manuscript were also limited by the constraints of the OPD, which can affect the generalizability of this study. To be listed on the OPD, an article author had to be a practicing clinician in the USA with an NPI number. The applicability of these results to other countries and scientists without an NPI number is unclear. While not every author of every manuscript examined in this study possessed Doctor of Medicine degrees, approximately $85 \%$ of the authors possessed NPI numbers, thereby meeting inclusion criteria for the OPD. An additional limitation may be that our clinician raters who provided article favorability ratings are admittedly not experts in each manuscript's specific field. However, we intentionally did not utilize experts within each specialty in order to better reflect the majority of manuscript readers, who are not experts, and to ensure the integrity of blinding. Experts may already be familiar with each abstract's authors and their COI due to preexisting knowledge of the field. Future studies could include assessing individual university databases of conflict of interest, interviewing published researchers on their perceptions of COIs and disclosure.

Acknowledgments: This work was supported by the Center for Clinical and Translational Sciences, which is funded by National Institutes of Health Clinical and Translational Award UL1 TROOO371 and KL2 TROOO37O from the National Center for Advancing Translational Sciences. Dr. Balentine is supported by a mentored career development award from the Agency for Healthcare Research and Quality (K12 HSO23009-03).

Corresponding Author: Karla Bernardi, MD; Department of Surgery General Surgery Clinical Research Fellow, Houston, TX, USA (e-mail: karla.bernardi@uth.tmc.edu).

Compliance with Ethical Standards

The content is solely the responsibility of the authors and does not necessarily represent the official views of the National Center for Research Resources or the National Institutes of Health.

Conflict of Interest: The authors declare that they do not have a conflict of interest.

Publisher's Note: Springer Nature remains neutral with regard to jurisdictional claims in published maps and institutional affiliations.

\section{REFERENCES}

1. C. D. DeAngelis, P. B. Fontanarosa, A. Flanagin, Reporting financial conflicts of interest and relationships between investigators and research sponsors. JAMA. 4, 286(1):89-91 (2001 Jul).

2. R. Steinbrook, Controlling conflict of interest-proposals from the Institute of Medicine. N Engl J Med. 360(21):2160-3 (2009 May 21).
3. O. Olavarria, H. L. Holihan, D. Cherla, C. A. Perez, L. S. Kao, T. C. Ko, M. K. Liang. A comparison of self-reported conflict of interest among published hernia researchers to the Centers of Medicare and Medicaid Services Open Payments Database. J Am Coll Surg. pii: S10727515(17)30136-9 (2017 Feb 3). [Epub ahead of print]

4. B. Lo, M. J. Field, Eds. Institute of Medicine. Conflict of Interest in Medical Research, Education and Practice (The National Academies Press, Washington, DC, 2009).

5. L. A. Bero, Q. Grundy. Why having a (nonfinancial) interest is not a conflict of interest. PLoS Biol. 14(12):e2001221 (2016 Dec 21).

6. S. Heres, J. Davis, K. Maino, E. Jetzinger, W. Kissling, S. Leucht. Why olanzapine beats risperidone, respiderone beats quetiapine, and quetiapine beats olanzapine: An exploratory analysis of head-to-head comparison studies of second-generation antipsychotics. Am J Psychiatry. 163(2):185-94 (2006 Feb).

7. M. E. Flacco, L. Manzoli, S. Boccia, L. Capasso, K. Aleksovska, A. Rosso, G. Scaioli, C. De Vito, R. Siliquini, P. Villari, J. P. Ioannidis. Head-to-head randomized trials are mostly industry sponsored and almost always favor the industry sponsor. J Clin Epidemiol. 68(7):81120 (2015 Jul).

8. Retraction Watch. A journal said it would retract a paper about asbestosnow it's "withdrawn" [Internet]. December 13, 2016. Available from: http://retractionwatch.com/category/by-reason-for-retraction/failureto-disclose-coi/

9. J. B. Hrachovec, M. Mora. Reporting of 6-month vs 12 -month data in a clinical trial of celecoxib. JAMA. 286(19):2398; author reply 2399-2400 (2001 Nov 21)

10. L. J. Hirsch. Conflicts of interest, authorship, and disclosures in industry-related scientific publications: The tort bar and editorial oversight of medical journals. Mayo Clin Proc. 84(9):811-21 (2009 Sep).

11. P. Doshi. No correction, no retraction, no apology, no comment: Paroxetine trial reanalysis raises questions about institutional responsibility. BMJ. 351:h4629 (2015 Sep 16).

12. Science 2.0. Conflict of interest? Ethics debate intensifies over retraction of flawed Séralini rat study [Internet]. Available from: http://www. science20.com/jon_entine_contrarian/conflict_interest_ethics_debate_ intensifies_over_retraction_flawed_s\%C3\%A9ralini_rat_study-127956

13. B. R. DeGeorge Jr, M. C. Holland, D. B. Drake. The impact of conflict of interact in abdominal wall reconstruction with acellular dermal matrix. Ann Plast Surg. 74(2):242-7 (2015 Feb).

14. Centers for Medicare and Medicaid Services. Open payments database. 2017. Available from: http://openpaymentsdata.cms.gov

15. E. Swanson. Textured breast implants, anaplastic large-cell lymphoma, and conflict of interest. Plast Reconstr Surg. 139(2):558e-559e (2017 Feb).

16. A. A. Jefferson, S. D. Pearson. Conflict of interest in Seminal Hepatitis C Virus and cholesterol management guidelines. JAMA Intern Med. 177(3):352-7 (2017 Mar 1).

17. J. P. Ioannidis. Why most published research findings are false. PLoS Med. 2(8):e124 (2005 Aug).

18. Equator Network. The strengthening the reporting of observational studies in epidemiology (STROBE) statement: Guidelines for reporting observational studies. January 2017. Available from: http://www.equator-network.org/reporting-guidelines/strobe/

19. Accreditation Council for Graduate Medical Education. January 2017. Available from: http://www.acgme.org/specialties

20. D. K. Flaherty DK. The vaccine-autism connection: A public health crisis caused by unethical medical practices and fraudulent science. Ann Pharmacother. 45(10): 1302-1304 (2011 Oct).

21. A. G. Dunn, D. Arachi, J. Hudgins, G. Tsafnat, E. Coiera, F. T. Bourgeois. Financial conflicts of interact and conclusions about neuraminidase inhibitors for influenza: An analysis of systematic reviews. Ann Intern Med. 161(7):513-8 (2014 Oct 7). 